\title{
Preliminary findings on the association between micronutrient deficiencies and under- or overnutrition: a systematic review
}

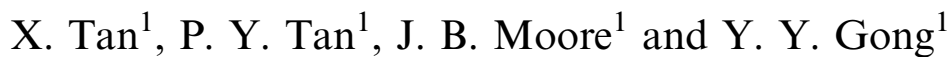 \\ ${ }^{1}$ School of Food Science and Nutrition, Faculty of Environment, University of Leeds, Leeds, UK
}

The double burden of malnutrition (DBM) refers to the coexistence of overnutrition (overweight and obesity) alongside undernutrition (stunting, wasting, underweight and micronutrient deficiencies) ${ }^{(1)}$. Deficiencies in micronutrients such as iron, zinc and vitamin A contribute to impaired immune function and poor growth and physical development in children ${ }^{(2)}$. While increasing evidence suggests micronutrient deficiencies are associated with overnutrition as well as undernutrition ${ }^{(3)}$, there are limited data summarising relevant magnitudes. Therefore, with the objective to establish if sufficient data exists to subject to meta-analyses, this study aimed to systematically review the available evidence on the associations between micronutrient status (zinc, iron and vitamin A) and under- or overnutrition in children and young people.

This systematic review was conducted by independent reviewers following PRISMA guidelines and prospectively registered at PROSPERO (CRD42020221523). The MEDLINE (Ovid), Scopus, Embase (Ovid) and Cochrane Library databases were searched from inception through February 1st 2021, for observational studies assessing micronutrient status (blood, serum or plasma levels of iron, zinc and vitamin A) and under- or overnutrition in young people ( $\leq 25$ years) of any ethnicity and gender. Article screening was managed using the web-based Rayyan software. Risk of bias assessment was conducted using the American Dietetic Association Quality Criteria Checklist.

Utilising comprehensive search strategies, a total of 7,830 articles were identified, and 4,570 articles remained after deduplication. After title and abstract screening, the full text of 266 articles were obtained to confirm eligible data for extraction. To date, 35 studies reporting associations between undernutrition or overnutrition and micronutrient status have been scrutinised; with some studies examining multiple micronutrients. Data extraction has been completed for 19 studies. Among these, 5 studies reported significant associations between overweight or obesity and alterations in micronutrient status: low serum iron $(\mathrm{OR}=1.78,1.34-2.37)$, iron deficiency $(\mathrm{OR}=3.96,1.34-11.67)$, low serum carotenoids $(\mathrm{OR}=2.51,1.43-4.39)$, and high serum retinol $(\mathrm{OR}=2.01$, 1.26-3.22 overweight; $\mathrm{OR}=2.90,1.65-5.09$ obesity) compared to normal weight. Seven studies reported significant correlations between micronutrient status and body mass index (BMI) as continuous variables. Positive associations were found for retinol $(\beta=5.56$, 3.36-7.75), blood haemoglobin $(r=0.121)$, plasma ferritin $(r=0.210)$, serum ferritin $(R 2=0.150 ; r=0.148$ for boys, $r=0.133$ for girls); and soluble transferrin receptor $(\mathrm{R} 2=0.058)$. Inverse associations were found for iron $(\beta=-0.870,-1.41$ to -0.329 for females, $\beta=-0.768,-1.31$ to -0.229 for males; $r=-0.057)$ and haemoglobin levels $(r=-0.055)$. A further seven studies found no significant associations between BMI and micronutrient levels.

In summary, while data extraction is ongoing we anticipate sufficient data are available to subject to meta-analyses. A better understanding of the associations between micronutrient status and under and overnutrition could contribute to the development of more effective strategies for micronutrient supplementation interventions in addressing DBM.

\section{References}

1. Popkin BM, Corvalan C \& Grummer-Strawn LM (2020) Lancet 395, 65-74

2. Bailey RL, West Jr. KP \& Black RE (2015) Ann Nutr Metab 66 (suppl 2), 22-33.

3. Kim M, Basharat A, Santosh R, et al. (2019) Diabetes Metab Res Rev 35, e3072. 